Tabellen und Abbildungen sind im Text zu kommentieren und mit einem Hinweis an der betreffenden Textstelle zu kennzeichnen. Bei Abbildungen und Tabellen aus anderen Publikationen ist die Quelle anzugeben. Umfangreiche Tabellen sind $\mathrm{zu}$ vermeiden.

Abbildungen und Tabellen sind mit Legenden $\mathrm{zu}$ versehen, fortlaufend $\mathrm{zu}$ numerieren und dem Manuskript auf separaten Blättern beizulegen (nicht in den Text einfügen). Die Legenden so abfassen, dass sie das Verständnis der Darstellung ohne Zuhilfenahme des Textes ermöglichen. In der Legende sind alle verwendeten Abkürzungen zu erläutern, soweit sie nicht schon in der Liste der Abkürzungen berücksichtigt sind.

Farbige Abbildungen sind erwünscht, wenn sie inhaltlich sinnvoll sind und eine ausreichende Druckqualität erzielt werden kann. Bei Einreichung per E-mail sind folgende Grafikformate möglich: tif, Auflösung 300 lpi; eps; jpeg. Falls diese Bedingungen technisch nicht erfüllt werden können oder die Dateien über $2 \mathrm{MB}$ gross werden, sollten Farbbilder in Form von Hochglanzkopien oder Dias an den Verlag gesandt werden; bei grafischen Darstellungen genügt auch ein einwandfreier Papierausdruck.
Für die Wiedergabe von bereits publizierten Abbildungen oder Tabellen ist eine Einwilligung des betreffenden Verlages notwendig.

Das Literaturverzeichnis ist in der Reihenfolge der Zitierung im Text und nicht alphabetisch zu ordnen und zu numerieren. Die entsprechende Nummer ist im Text in Klammern anzuführen.

Die ersten sechs Autorennamen und die vollständigen Titel der zitierten Arbeiten sind zu nennen. Bei mehr als sechs Autoren "et al.» hinzufügen.

\section{Beispiele:}

1 Vega KJ, Pina I, Krevsky B. Heart transplantation is associated with an increased risk for pancreatobiliary disease. Ann Intern Med 1996;124:980-3.

2 Parkin DM, Clayton D, Black RJ, Masyer E, Friedl HP, Ivanov E, et al. Childhood-leukaemia in Europe after Chernobyl: 5-year follow-up. Br J Cancer 1996;73:1006-12.

3 Ringsven MK, Bond D. Gerontology and leadership skills for nurses. 2nd ed. Albany (NY): Delmar Publishers; 1996.

4 Philips SJ, Whisnant JP. Hypertension and stroke. In: Laragh JH, Brenner BM, eds. Hypertension: pathophysiology, diagnosis, and management. 2nd ed. New York: Raven Press; 1995. p. 465-78.

\title{
Directives pour les auteurs du Forum Médical Suisse
}

Le Forum Médical Suisse publie des articles contribuant à la formation continue touchant à toutes les disciplines de la médecine. Les directives pour les auteurs suivent les recommandations de l'«International Committee of Medical Journal Editors» (Uniform requirements for manuscripts submitted to biomedical journals. JAMA 1997;277:92734).

Les articles des rubriques "Editorial», "Le cas particulier", "Coup d'œil", "Erreurs et errances " peuvent être remis spontanément. Les articles se rapportant à la formation permanente sont en général à l'initiative de la rédaction.

Les manuscrits des rubriques "Curriculum" et "Cabinet" sont soumis à la rédaction et à des experts externes («reviewers»).

\section{Remise des manuscrits}

Les articles peuvent êtres rédigés en langue française ou allemande.
La lettre d'accompagnement doit être signée par tous les auteurs. Ils certifient ainsi qu'ils ont lu, vérifié et approuvé le manuscrit.

Les auteurs y indiqueront les éventuelles obligations financières ou personnelles qui ont été conclues en rapport avec l'article soumis. Des contributions financières autres que celles apportées par l'institution d'origine sont à mentionner dans une annotation de l'article, de même que d'autres relations financières ou personnelles en rapport avec le manuscrit.

Le manuscrit intégral doit être remis à la rédaction, autant que possible par e-mail (voir l'adresse ci-dessous).

\section{Rubriques, contenu, longueur}

Formation continue

Curriculum: Les articles de la rubrique "Curriculum" passent en revue les thèmes principaux de la médecine interne selon un roulement de trois ans. Composition: intro-
Detaillierte Anweisungen mit zahlreichen Beispielen sind in den "Uniform Requirements" enthalten:http://jama.ama-assn.org/ info/auinst_req.html

\section{Adresse des Verlages}

Bitte alle Manuskripte an folgende Adresse einsenden:

EMH Schweizerischer Ärzteverlag AG

Redaktionssekretariat SMF

Postfach

4010 Basel

Tel. 061 / 4678552

Fax $061 / 4678556$

E-mail: smf@emh.ch

Internet: http://www.emh.ch

duction générale et conclusion de synthèse, partie centrale se rapportant aux connaissances médicales les plus récentes sur le sujet. Longueur maximum 8 pages de manuscrit (soit 17000 caractères environ); en principe, article sur demande.

Cabinet: Les articles de la rubrique "Cabinet" doivent traiter des thèmes principaux touchant au diagnostic des médecins exerçant en cabinet et aux traitements prescrits. Les thèmes sont planifiés sur trois ans. Ces articles doivent tenir compte de la situation particulière du médecin en cabinet, des moyens techniques et $\mathrm{du}$ facteur temps. Orientation: du symptôme au diagnostic. Longueur maximum: 8 pages de manuscrit (soit 17000 caractères environ); en principe, article sur demande.

Les articles des rubriques "Curriculum" et "Cabinet" doivent traiter des thèmes choisis en s'attachant à leurs aspects dans la pratique et aux applications possibles. Ils ne doivent mentionner que les conclusions les plus importantes. Ils doivent aussi avoir un lien avec les directives (en particulier celles approuvées par la FMH), sans les reprendre obligatoirement. Les analyses de coût et les réflexions de rapport coût/utilité sont des éléments informatifs importants de ces articles.

\section{Observations de cas}

Le FMS peut publier les observations de cas sous différentes formes: présentation dans 
la rubrique "Coup d'œil", sous forme de lettre à la rédaction ou, lorsque l'article présente un grand intérêt, sous le titre "Le cas particulier».

Toute indication permettant d'identifier des patients ne figurera dans le manuscrit que si elle sert le contenu scientifique de celuici et uniquement après consentement écrit du patient («Informed consent»; aussi pour les illustrations). Il est interdit de modifier les indications relatives aux patients afin de préserver l'anonymat de ces derniers.

Les publications dont le contenu recoupe celui du travail soumis seront jointes au manuscrit.

Le cas particulier: En publiant des casuistiques, le FMS vise, en se basant sur l'observation de cas isolés ou peu nombreux, à favoriser l'engramme du médecin, à augmenter l'acuité du diagnostic, à éveiller des associations. L'article (en français ou en allemand) ne doit pas dépasser 8500 caractères (texte seulement), respectivement 7500 caractères (avec une illustration au maximum). Il s'y ajoute un titre (concis et percutant), les noms des auteurs (avec indication de leur institution d'origine), une adresse pour la correspondance et 5 références bibliographiques au maximum. Composition: pas de résumé, d'introduction ni de sous-titre. L'article commence d'emblée avec la description $\mathrm{du}$ cas. Les résultats d'examen et le déroulement sont intégrés au texte, exceptionnellement sous forme de tableau. La description du cas et le diagnostic sont suivis d'un bref commentaire (signification épidémiologique et clinique; évt. problématique particulière, état actuel de la science, nouvelles découvertes, aspects thérapeutiques).

Coup d'œil: les faits remarquables de toutes les disciplines de la médecine. Des images, illustrations et représentations graphiques peuvent être remis à la rédaction accompagnées du titre, nom de l'auteur et un texte explicatif (1/2 page au maximum).

Lettres à la rédaction: Le FMS favorise l'échange d'opinions, grâce au courrier des lecteurs, qu'il s'agisse de commentaires ou des publications précédentes ou de missives spontanées. Les lettres ne doivent pas dépasser 1800 caractères. Elles peuvent également soulever des questions concrètes, dans la mesure où il est possible de leur donner réponse dans un espace aussi réduit.

\section{Autres rubriques}

Editoriaux sur des thèmes actuels. Ils ne doivent pas dépasser une longueur de 2 pages. Recherche.ch: Etat actuel de la recherche en Suisse. En principe, article sur demande ou en accord avec la rédaction.
Erreurs et errances médicales: Tours d'horizon de controverses concernant des thérapies anciennes mais jamais prouvées et sur des errances médicales.

Nova: nouveaux diagnostics, nouvelles thérapies. Articles sur demande.

Périscope: résumés de publications cliniques. Articles coordonnés par la rédaction.

Biomédecine.com: publications de base se rapportant à des problèmes cliniques actuels. Articles coordonnés par la rédaction. Traits de lumière: publication de fin d'année. Articles sur demande.

\section{Orthographe et présentation du manuscrit}

Les abréviations courantes (par ex. ACTH, EEG) sont admises. Toutes les autres abréviations devront être employées de manière restrictive; elles sont à expliciter dans une liste alphabétique. Les abréviations utilisées une seule fois sont à éviter. Par principe, n'employer que des unités SI. Des résultats exprimés au moyen d'autres unités peuvent être cités, le cas échéant, entre parenthèses. Les tableaux et les figures doivent être commentés dans le texte et mentionnés dans le paragraphe correspondant. Lors d'utilisation de figures et de tableaux provenant d'autres publications, leur source est à indiquer. Des tableaux trop chargés sont à éviter

Les figures et les tableaux seront accompagnés de légendes, numérotés en continu et annexés sur feuilles séparées au manuscrit. $\mathrm{Ne}$ pas les intégrer au texte. Les légendes doivent être rédigées de manière à expliciter la figure sans qu'un recours au texte ne s'impose. Dans la légende, toutes les abréviations sont à expliciter si elles n'ont pas déjà été mentionnées sur la liste des abréviations.

Des reproductions en couleurs sont bienvenues si leur valeur illustrative et leur qualité d'impression sont d'un niveau satisfaisant. Pour les envois par e-mail, les formats suivants sont possibles: tif, définition 300 lpi; eps; jpeg: Si ces conditions techniques ne peuvent être remplies ou si les fichiers dépassent une taille de $2 \mathrm{MB}$, les illustrations en couleurs peuvent être adressées à la rédaction sous forme de photographies (sur papier brillant) ou de diapositives. Pour les représentations graphiques, d'impeccables reproductions sur papier suffisent.
La reproduction d'illustrations ou de tableaux déjà publiés requiert le consentement de la maison d'édition concernée. L'index bibliographique doit suivre l'ordre des citations dans le texte (et non l'ordre alphabétique) et doit être numéroté de même manière. Le numéro correspondant doit figurer entre parenthèses dans le texte.

Les noms des six premiers auteurs ainsi que les titres complets de tous les travaux cités doivent être indiqués. S'il y a plus de six auteurs, il faut ajouter la mention «et al.».

\section{Exemples:}

1 Vega KJ, Pina I, Krevsky B. Heart transplantation is associated with an increased risk for pancreatobiliary disease. Ann Intern Med 1996;124:980-3.

2 Parkin DM, Clayton D, Black RJ, Masyer E, Friedl HP, Ivanov E, et al. Childhood-leukaemia in Europe after Chernobyl: 5-year follow-up. Br J Cancer 1996;73: 1006-12.

3 Ringsven MK, Bond D. Gerontology and leadership skills for nurses. 2nd ed. Albany (NY): Delmar Publishers; 1996.

4 Philips SJ, Whisnant JP. Hypertension and stroke. In: Laragh JH, Brenner BM, eds. Hypertension: pathophysiology, diagnosis, and management. 2nd ed. New York: Raven Press; 1995. p. 465-78.

Des informations détaillées avec de nombreux exemples sont contenues dans les "Uniform Requirements" à l'adresse suivante: http://jama.ama-assn.org/info/ auinst_req.html

\section{Adresse de la maison d'édition}

Veuillez adresser tous les manuscrits à l'adresse suivante:

EMH Editions médicales suisses SA Secrétariat de rédaction FMS

Case postale

4010 Bâle

tél. 0614678552

fax 0614678556

e-mail: smf@emh.ch

Internet: http://www.emh.ch 